ARTÍCULOS DE INVESTIGACIÓN

\title{
Aplicación del método de evaluación basada en la certeza en la enseñanza del derecho: Un estudio exploratorio en alumnos de primer año de la carrera de Derecho
}

\author{
Aplicação do método de avaliação com base na certeza no ensino de direito: \\ um estudo exploratório com estudantes de direito do primeiro ano
}

\section{Application of the certainty-based marking method in legal education: an exploratory study in first-year law students}

\author{
Sulan Wong \\ Universidad de La Frontera, Chile
}

Julio Rojas-Mora

Universidad Católica de Temuco, Chile

\begin{abstract}
RESUMEN Como estrategia disruptiva de la tradición «bancaria» de la enseñanza del derecho, en este trabajo se expone la aplicación del método de evaluación basada en la certeza en un curso obligatorio de primer año de la carrera de Derecho de una universidad del sur de Chile durante 2018 y 2019. Mediante este método se busca pasar del mero «recuerdo» de conocimientos a la «evaluación» de los mismos. Para lograr este objetivo, a través un modelo de aprendizaje autorregulado, los estudiantes deben autoevaluarse con retroalimentación interna y externa. Los resultados principales estadísticos alcanzados, mediante una prueba binomial exacta, muestran que los alumnos aplican procesos de autorregulación y autoevaluación al seleccionar el nivel de certeza que maximiza su puntaje esperado. Asimismo, mediante un modelo logístico mixto con efectos aleatorios, controlando por efectos fijos de prueba, podemos confirmar que la probabilidad de responder correctamente una pregunta tiene como predictores significativos la retroalimentación, la extensión de la prueba y el nivel de certeza de la respuesta. De manera contraria, el género del estudiante no es un predictor significativo, ni aisladamente, ni como término de interacción con otras covariables.
\end{abstract}


PALABRAS CLAVE Educación en derecho, metacognición, autorregulación, autoevaluación, retroalimentación, diferencias de género.

RESUMO Como estratégia disruptiva da tradição «bancária» do ensino do direito, este artigo apresenta a aplicação do método de Avaliação Baseada em Certeza (CBM) em uma disciplina obrigatória do primeiro ano da graduação em direito de uma universidade do sul do Chile, durante o ano letivo 2018-2019. A CBM busca passar da mera «memória» do conhecimento para a «avaliação» do mesmo. Para atingir esse objetivo, as/os estudantes, através de um modelo de aprendizagem autorregulado, devem se autoavaliar com retroalimentação interna e externa. Os principais resultados estatísticos alcançados, usando um teste binomial exato, mostram que as/os estudantes aplicam processos de autorregulação e autoavaliação ao selecionar o nível de certeza que maximiza sua pontuação esperada. Assim, mesmo, usando um modelo logístico misto com efeitos aleatórios, controlando os efeitos fixos da prova, podemos confirmar que a probabilidade de responder corretamente a uma pergunta tem a retroalimentação, a extensão da prova e o nível de certeza da resposta como preditores significativos. Por outro lado, o sexo do/da estudante não é um preditor significativo, nem isoladamente nem como termo de interação com outras covariáveis.

PALAVRAS-CHAVE Educação jurídica, meta-cognição, autorregulação, autoavaliação, comentários, diferenças de gênero.

ABSTRACT As a disruptive strategy to the «banking» tradition of legal education, this work presents the application of the Certainty-Based Marking (CBM) method in a compulsory first-year course of the law degree of a university in southern Chile during the 2018- 2019 academic year. CBM seeks to move from merely «remembering» knowledge to its «evaluation». Students must achieve this goal through a self-regulated learning (SRL) model. They should self-evaluate themselves through both internal and external feedback. The main statistic results obtained with an exact binomial test show that students apply both self-regulation and self-evaluation while selecting the level of confidence that maximizes their expected score. Likewise, through a mixed logistic model with random effects, controlling for fixed exam effects, there is confirmation that the probability of correctly responding questions has significant predictors such as feedback, the exam's length, and the level of certainty in the answer. In contrast, the student's gender is not a significant predictor, neither in isolation nor as an interaction term with other covariates.

KEYWORDS Legal education, metacognition, self-regulation, self-evaluation, feedback, gender differences. 


\section{Introducción}

La enseñanza del derecho se ha centrado en el modelo «catedrático» o «bancario»(Freire, 2018) con el que el docente hace clases expositivas en las que los estudiantes participan de manera limitada, ya que su actividad principal consiste en una transcripción verbatim de lo que el profesor expone a medida que avanza la clase, intentando, en su razonamiento, asegurar una calificación aprobatoria al repetir los contenidos durante las evaluaciones subsiguientes. Ya en 1997, en una crítica a la enseñanza del derecho en Chile, el profesor Roberto Guerrero (1997) advirtió que se había llegado al extremo de registrar las clases a través de dispositivos electrónicos con el objetivo de garantizar, con la mayor fidelidad posible, lo que el profesor había dicho en sus clases. En nuestra experiencia, cuando por motivos metodológicos el docente impide la grabación de las sesiones, los estudiantes acuerdan la toma simultánea de apuntes para luego hacer un compendium que dé cuenta fielmente de las mismas. Como el zorro persiguiéndose la cola, los docentes, generadores y receptores de esta dinámica, la refuerzan al asumir la posición más cómoda, constatando mediante sus estrategias de evaluación escrita u oral el nivel de retención y, por ende, de repetición fidedigna de los contenidos asociados al temario de una asignatura (Guerrero, 1997; Squella Narducci, 2000; Rodríguez Ferrara, 2004; Coloma Correa, 2005; Fernández-Costales Muñiz, 2014).

En este modelo de enseñanza, los procesos metacognitivos se encuentran ausentes. Mediante la metacognición, son nuestros propios pensamientos el objeto de nuestro pensamiento, y la mejor forma acceder a ellos es, paradójicamente, a través del diálogo (Amsterdam y Bruner, 2000: 237). Por tanto, una sesión de clases en la que la interacción y la participación de los estudiantes es limitada impide la utilización de procesos metacognitivos de evaluación. En este sentido, McDonald y otros (2000) exponen que cuando «la evaluación de los estudiantes se concentra sobre las materias que son fáciles de evaluar, se conduce a un énfasis exagerado en la memorización y en la obtención de habilidades de nivel más bajo». Esta conclusión se condice con el hecho de que el mero recuerdo corresponde al nivel cognitivo más bajo de la taxonomía de Bloom, modificada por Anderson y Krathwohl (2001: 31), mientras que la metacognición requiere de evaluación, el segundo más alto de la misma. Si bien es cierto que para evaluar (desde la perspectiva del estudiante) hay que recordar, la evaluación no debería centrarse en el segundo proceso (desde la perspectiva del docente), sino que debería lograrse el continuum cognitivo que los une (recordar, comprender, aplicar, analizar y evaluar). Como parte de los procesos metacognitivos, concordamos con McDonald y otros (2001: 47), quienes mencionan que la capacidad de autoevaluarse es para los estudiantes más valiosa que el aprendizaje de una materia en particular, ya que «necesitan ser capaces de hacer juicios confiables acerca de lo que saben y lo que no, y de lo que pueden y no pueden hacer». Coloma (2005: 25), 
entre otros autores abocados a los estudios pedagógicos en el derecho, expone que la aplicación de procesos de autoevaluación entre los estudiantes es una herramienta que podría contribuir al desarrollo de una actitud que les ayude a afrontar sus tareas profesionales de un modo autocrítico.

La autoevaluación es una competencia autorregulatoria que incide positivamente en la adecuada selección e interpretación de manera que provea retroalimentación. De acuerdo con Paris y Winograd (1990), la autoevaluación se compone de dos aspectos principales. En primer lugar, se encuentra la autovaloración, el juicio acerca de las capacidades cognitivas personales, factores de las tareas que influencian la dificultad cognitiva, o estrategias cognitivas que pueden facilitar o impedir la realización de la tarea. En segundo lugar, se encuentra la autogestión, la forma en la que la metacognición ayuda a organizar los aspectos cognitivos de la realización de una tarea. Estudiantes que logran un alto nivel de autovaloración y autogestión serán capaces de enfrentarse a problemas complejos, identificando fallas de conocimiento y la manera en que afectan estas la forma de enfrentarse a los mismos.

Podemos equiparar esta definición de «autoevaluación» con lo que la literatura pedagógica contemporánea, de la mano de autores como Butler y Winne (1995), ha denominado como modelo de aprendizaje autorregulado (Self-Regulated Learning), es decir, el establecimiento de estrategias para que el estudiante pueda conocer profundamente la calidad de su conocimiento, sus creencias, sus motivaciones y sus procesos cognitivos. Para estos autores, la retroalimentación es el proceso que permite reducir la discrepancia entre la comprensión actual que el estudiante tiene y la que se desea que tenga. No obstante, hay que recordar que aunque los reglamentos académicos y los procesos de acreditación insisten en la importancia de la evaluación con una rúbrica o pauta clara y objetiva, es poco frecuente su uso, y entre aquellos docentes que lo hacen existen quienes lo implementan de manera inadecuada. Esto último tal vez se debe a la dificultad de concretar en un instrumento de evaluación objetiva el proceso subjetivo que el docente implementa, algo que en poco o nada contribuye a la retroalimentación.

De acuerdo con Hattie y Timperley (2007), la retroalimentación, como proceso fundamental del modelo Self-Regulated Learning, busca impactar en cuatro niveles: i) el de tarea, cuando el estudiante recibe información acerca de qué tan bien ha comprendido y ejecutado la misma; ii) el de proceso, cuando el estudiante recibe información acerca del proceso principal para comprender y ejecutar una tarea; iii) el de autorregulación, cuando el estudiante monitorea, dirige y regula sus acciones; $y$ iv) el personal, cuando el estudiante recibe evaluaciones personales de carácter usualmente positivo.

En este artículo presentamos los resultados de la aplicación del método de evaluación basado en la certeza (Certainty-Based Marking) en un curso de primer año de la carrera de Derecho de una universidad del sur de Chile durante 2018 y 2019. Este 
método fue desarrollado por Gardner-Medwin (1995) para inducir a los estudiantes a la autorregulación y a la reflexión cuidadosa sobre las preguntas en pruebas objetivas. Como objetivo fundamental de la aplicación del método Certainty-Based Marking y de este trabajo, buscamos entender si los estudiantes son capaces de realizar el proceso de autorregulación necesario para estimar adecuadamente la probabilidad de responder correctamente a una pregunta dada. Asimismo, queremos observar si los procesos de retroalimentación y la evaluación acumulativa, como lo establece la literatura, permiten mejorar este proceso. Finalmente, incluimos el género de los estudiantes como variable autónoma de control y de interacción, ya que existe mucha literatura que aborda su efecto en el rendimiento alcanzado por individuos en ambientes competitivos y cooperativos.

\section{Marco teórico}

\section{Modelo de aprendizaje autorregulado}

La mayoría de las definiciones sobre aprendizaje autorregulado, según Zimmerman (1989), varían de acuerdo con la perspectiva teórica de los investigadores, pero se pueden sistematizar alrededor de tres características fundamentales. En primer lugar, estas coinciden en establecer un uso intencional de procesos, estrategias o respuestas específicas de parte de los estudiantes para mejorar sus objetivos académicos. En segundo lugar, las definiciones sobre el modelo Self-Regulated Learning se caracterizan por enfocarse en el ciclo continuo de retroalimentación autorregulada. En este ciclo, los estudiantes monitorean la efectividad de sus propios métodos o estrategias de aprendizaje, lo que les permite, por ejemplo, modificarlas o reemplazarlas en función de sus objetivos de aprendizaje. En tercer lugar, el autor encuentra que las definiciones sobre el modelo Self-Regulated Learning se preocupan por describir cómo y por qué los estudiantes deciden usar, especialmente, un proceso, estrategia o respuesta de autorregulación en lugar de otro.

El modelo Self-Regulated Learning de Butler y Winne (1995) se conceptualiza sobre la base de una serie de episodios volitivos que en su conjunto son caracterizados por un flujo cíclico de información: con la asignación de una tarea, los estudiantes recurren a sus conocimientos y sus creencias para construir una interpretación de los requerimientos y propiedades de ella. Sobre la base de la interpretación construida, se proponen objetivos que buscan lograr, aplicando estrategias y tácticas para generar los productos requeridos. Estas estrategias y prácticas pueden ser tanto mentales (cognitivas o afectivas/emocionales) como conductuales. El monitoreo del proceso de participación en la tarea realizado por el estudiante y la actualización de los productos genera, progresivamente, una retroalimentación interna. La información entregada en la retroalimentación aporta bases que permiten a los estudiantes rein- 
terpretar elementos de la tarea y su participación en la misma, dirigiendo, de esta manera, el compromiso subsiguiente. En este proceso interno, los estudiantes pueden modificar su participación definiendo nuevos objetivos o ajustando los existentes: los estudiantes pueden reexaminar sus tácticas y estrategias, seleccionar aproximaciones más productivas a la tarea, adaptar sus habilidades e incluso, algunas veces, generar nuevos procedimientos. Si se realiza un proceso de retroalimentación externa, esa información adicional le permite a los estudiantes confirmar, mejorar o contradecir las interpretaciones de la tarea realizada por el estudiante y el proceso llevado a cabo para realizarla. Por lo tanto, y como resultado del monitoreo de su participación en la tarea, los estudiantes modificarán sus creencias y conocimientos, lo que incidirá en una subsecuente autorregulación. Según Hattie y Timperley (2007), la autorregulación implica autonomía, autocontrol, autodirección y autodisciplina, mientras que la retroalimentación implica aporte de información como «consecuencia» del rendimiento o comprensión del estudiante. Así, por ejemplo, el profesor puede dar información correctiva, un "par» puede indicar la estrategia más conveniente a seguir y un libro puede entregar información para complementar o aclarar las ideas. A partir de la información obtenida, el estudiante puede completar, aclarar, corregir, restructurar o confirmar el "grado de confianza» que tiene sobre las afirmaciones que realiza (Hattie y Timperley, 2007: 94). Podemos interpretar que la retroalimentación, en este sentido, deja de ser puramente información correctiva y se transforma en una nueva instrucción que reduce la distancia entre el rendimiento y el objetivo de aprendizaje deseado, o entre el grado de confianza que sobre una afirmación tiene un estudiante y la probabilidad de responder correctamente a una pregunta: los estudiantes menos efectivos tienen estrategias de autorregulación mínimas y dependen mucho más de factores externos, como el docente o la tarea, para obtener la retroalimentación necesaria para alcanzar este objetivo (Hattie y Timperley, 2007: 94). El grado de confianza que los estudiantes tienen en la veracidad de sus respuestas, explican Hattie y Timperley, puede afectar la receptividad y la búsqueda de la retroalimentación, que tiene su mayor efecto cuando el estudiante espera una respuesta correcta que resulta incorrecta (Hattie y Timperley, 2007: 95). Como veremos en la siguiente sección, el método Certainty-Based Marking busca identificar situaciones como estas para mejorar el proceso de aprendizaje.

\section{Certainty-Based Marking}

El método Certainty-Based Marking fue introducido en evaluaciones formativas de cursos de las escuelas de Medicina de la ciudad de Londres, ${ }^{1}$ y desde 2001 ha sido aplicado en pruebas sumativas en la University City London, donde ha sido considerado por los estudiantes como simple, justo, fácilmente comprensible y beneficioso, ya que les motiva a justificar, en un proceso de autoregulación, las razones que les llevan a 
tener o no certeza en su respuesta (Gardner-Medwin, 2006: 141-149).

Dicho método consiste en una sencilla modificación a la tradicional fórmula de "corrección por adivinación», en la que una cantidad de preguntas correctamente respondidas son eliminadas por cada pregunta incorrectamente respondida (Thurstone, 1919). En este caso, el estudiante no solo debe seleccionar la respuesta correcta, sino que además debe establecer el nivel de certeza que tiene en la misma. De acuerdo con la combinación certeza-resultado, se asigna una puntuación positiva cuando la respuesta es correcta o negativa cuando no lo es. ${ }^{2}$

A partir del «grado de certeza» que tiene un estudiante acerca de una afirmación verdadera, Gardner-Medwin y Gahan (2003) exponen que se encuentra en uno de estos cinco estados:

- De conocimiento: $\mu=1$

- De incertidumbre: $0,5<\mu<1$

- De ignorancia: $\mu=0,5$

- De conocimiento erróneamente fundado: $0<\mu<0,5$

- De engaño: $\mu=0$

La idea subyacente en el método Certainty-Based Marking es ayudar a los estudiantes a ubicarse en esta escala mediante un proceso reflexivo y metacognitivo, es decir, autorregulado. Un estudiante debe ser capaz de identificar adecuadamente la probabilidad de responder correctamente, para así poder escoger la certeza que maximice su puntuación esperada. ${ }^{3}$ Como Gardner-Medwin (2019) expone, la certeza es una forma de pronóstico de probabilidad, es decir, un estimador de la probabilidad de que una respuesta sea correcta.

Un tema que queda por discutir en esta sección es si el establecimiento de incentivos diferenciados, definidos por el esquema de puntuación basado en la combinación certeza-resultado, es neutral con respecto al género de los estudiantes. Existe una gran variedad de estudios en los que se establecen diferencias debido al género en relación con el establecimiento de incentivos y a los comportamientos en ambientes competitivos. Gneezy, Niederle y Rustichini (2003) encontraron que en ambientes competitivos mixtos (con participación de hombres y mujeres), los incentivos esta-

2. Aclararemos el esquema de puntuación seleccionado para cada combinación certeza-resultado en la sección «Método».

3. Por tanto, el «grado de certeza» y el original «grado de creencia» son también equivalentes en este trabajo al concepto de "probabilidad subjetiva», como la concepción clásica lo ha hecho. No obstante, existen discrepancias al respecto sobre las que no ahondaremos, cuando la «vaguedad» suplanta a la «incertidumbre». Recomendamos al lector interesado en el tema revisar los aportes propuestos en Dietz y Sebastiano (2009) y en Smith (2008). 
blecidos crean una disparidad en el rendimiento de hombres sobre el de las mujeres. La hipótesis de diferencia en el género se prueba, ya que al introducir los mismos incentivos a la competencia en ambientes monogenéricos (conformados únicamente por hombres o por mujeres), el rendimiento promedio de las mujeres mejora, mientras la diferencia con el rendimiento promedio de los hombres se reduce. Por su parte, en su estudio basado en trabajos grupales de estudiantes de educación superior de ciencias e ingeniería, Martinho, Albergaria-Almeida y Dias (2015) hallaron que las mujeres favorecen la cooperación, mientras que los hombres prefieren la competencia. Morin (2015), por su parte, estudió la abolición del decimotercer año de estudio en Ottawa en 1997, lo que produjo una doble cohorte de postulantes a las universidades de la región y una sobredemanda de acceso a las mismas; el incremento exógeno de la competencia impactó sobre la calidad de los estudiantes aceptados y, a la larga, en el desempeño de los mismos, lo que produjo un mayor impacto positivo en las tasas de titulación oportuna de los hombres sobre el de las mujeres. También, Levitt y otros (2016) han encontrado diferencias relacionadas al género en el sistema educativo al determinar que los niños tienden a ser más sensibles a incentivos de corto plazo (halago, retroalimentación positiva e incentivos explícitos) que las niñas, mientras que estas lo son a los incentivos de largo plazo (formación de capital humano). El trabajo de Guinier, Fine y Balin (1994), adecuadamente titulado «Becoming Gentleman: Women's Experiences at One Ivy League Law School», ha sido la semilla de un larga línea de investigación sobre las diferencias de género en las escuelas de Derecho estadounidenses. Sus autoras argumentan que las diferencias generadas en el primer año y mantenidas en los restantes tres años de la carrera, hacen que la probabilidad de pertenecer al 10\% superior de sus respectivas cohortes sea tres veces mayor para hombres que para mujeres. En el ejercicio de la abogacía, Azmat y Ferrer (2017), con datos estadounidenses, encuentran grandes brechas en el rendimiento de los abogados jóvenes desde la perspectiva del número de horas facturadas (10\% más) y del número de clientes conseguidos (cerca del doble), lo que lógicamente lleva a una brecha salarial. Aunque la presencia de hijos pequeños en el hogar es uno de los factores más importantes en la explicación del fenómeno, las aspiraciones de los individuos — su aspecto competitivo- también juegan un papel significativo en el mismo.

A partir de esta evidencia, es de esperarse que en un entorno competitivo, como es la evaluación en un curso, pudiese verse alguna diferencia entre los géneros. Sin embargo, Gardner-Medwin y Gahan (2006) no encontraron diferencias significativas en el rendimiento debidas al género de los estudiantes al aplicar el método CertaintyBased Marking. Por esta razón, decidimos incluir la variable de género en nuestro estudio.

\section{Método}


Tabla 1. Esquema de puntuación utilizado.

\begin{tabular}{|lccc|}
\hline & Certeza baja & Certeza media & Certeza alta \\
Resultado correcto & 1 & 2 & 3 \\
Resultado incorrecto & 0 & -1 & -4 \\
\hline
\end{tabular}

Fuente: Gardner-Medwin (2006).

Se recogieron los resultados de las cinco pruebas del método Certainty-Based Marking sumativas y acumulativas ${ }^{4}$ en un curso de primer año de la carrera de Derecho de una universidad del sur de Chile durante 2018 y 2019. En la tabla 1 se presentan las puntuaciones asignadas a cada combinación certeza-resultado, siguiendo la recomendación de Gardner-Medwin (2006) para preguntas de selección múltiple (cuatro posibles respuestas por cada pregunta).

Después de corregida cada prueba, y como principal herramienta pedagógica, se realiza un proceso de retroalimentación dentro de las dos semanas siguientes de su aplicación, el que se inicia con la entrega de la prueba corregida a todos los estudiantes en la sala de clases.

Cada estudiante tiene la oportunidad de revisarla de manera individual para verificar el puntaje obtenido de acuerdo con sus aciertos y desaciertos. Se revisan cada una de las preguntas de la prueba de manera grupal, animando a los estudiantes a debatir entre ellos sobre la justificación que los llevó a responder de una manera determinada y con el nivel de certeza seleccionado. El debate en la sala de clases puede llegar a crear cierta tensión entre quienes tienen la respuesta correcta y quienes creen tenerla. En ese momento, el docente que guía el debate identifica y corrige tanto los errores y las ideas equivocadas subyacentes en las justificaciones que llevaron a responder incorrectamente, como a responder correctamente sobre una justificación incorrecta. Además, se le recomienda a los estudiantes revisar de nuevo algunos textos para complementar la información faltante. Con la información entregada los estudiantes pueden, como sostienen Hattie y Timperley (2007), completar, aclarar, corregir, reestructurar o confirmar el «grado de verdad» que tienen sobre las afirmaciones que realizan. La información entregada no es solamente correctiva, sino que en realidad se transforma en una nueva instrucción en el proceso de aprendizaje que ayuda a reducir la diferencia entre el rendimiento alcanzado y el objetivo de aprendizaje deseado. Sin embargo, la retroalimentación no genera una nueva instrucción si el estudiante no inicia, a partir de la misma y de manera autónoma, consciente e independiente, el proceso de aprendizaje que genere un compromiso subsiguiente. Este compromiso autodirigido en la modificación del aprendizaje se verifica en la prueba siguiente que, al ser acumulativa, permite verificar el proceso de autorregulación de

4. Cada una de las pruebas realizadas incluye los contenidos evaluados en las anteriores. 
Tabla 2. Resumen de evaluaciones

\begin{tabular}{|c|c|c|c|c|c|c|}
\hline & Prueba & 1 & 2 & 3 & 4 & 5 \\
\hline & Preguntas & 15 & 25 & 25 & 18 & 22 \\
\hline & $\begin{array}{l}\text { Estudiantes } \\
\text { (Fem./Masc.) }\end{array}$ & $\begin{array}{c}36 \\
(24 / 12)\end{array}$ & $\begin{array}{c}36 \\
(24 / 12)\end{array}$ & $\begin{array}{c}34 \\
(23 / 11)\end{array}$ & $\begin{array}{c}28 \\
(17 / 11)\end{array}$ & $\begin{array}{c}29 \\
(18 / 11)\end{array}$ \\
\hline \multirow{6}{*}{ 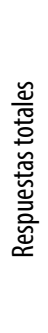 } & Alta-Correcta & $208 / 38,7 \%$ & $353 / 39,3 \%$ & $354 / 41,8 \%$ & $246 / 49,0 \%$ & $324 / 50,9 \%$ \\
\hline & Alta-Incorrecta & $68 / 12,6 \%$ & $56 / 6,2 \%$ & $56 / 6,2 \%$ & $44 / 8,8 \%$ & $20 / 3,1 \%$ \\
\hline & Media-Correcta & $88 / 16.4 \%$ & $169 / 18,9 \%$ & $180 / 21,3 \%$ & $83 / 16,5 \%$ & $101 / 15,9 \%$ \\
\hline & Media-Incorrecta & $90 / 16,7 \%$ & $106 / 11,8 \%$ & $103 / 12,2 \%$ & $51 / 10,2 \%$ & $57 / 8,9 \%$ \\
\hline & Baja-Correcta & $34 / 6,3 \%$ & $97 / 10,8 \%$ & $77 / 9,1 \%$ & $36 / 7,2 \%$ & $63 / 9,9 \%$ \\
\hline & Baja-Incorrecta & $50 / 9,3 \%$ & $117 / 13,0 \%$ & $87 / 10,3 \%$ & $42 / 8,8 \%$ & $72 / 11,3 \%$ \\
\hline
\end{tabular}

Fuente: Elaboración propia.

los estudiantes.

Estadísticamente procedimos a analizar los datos en dos frentes. En primer lugar, y de acuerdo con la descripción teórica desarrollada por Gardner-Medwin (1995), verificamos que el nivel de certeza seleccionado por los estudiantes sea el adecuado para maximizar su resultado esperado. Calculando la proporción de respuestas correctas y los intervalos de confianza $(\alpha=0,05)$ para todas las respuestas obtenidas, se puede estimar qué tan adecuadas lo han sido con respecto a los puntos de corte teóricos que se obtienen de la tabla 1.

El segundo frente de análisis estadístico consistió en un modelo de regresión logística de efectos fijos, en primer término, y un modelo de regresión logística mixto con efectos aleatorios, controlando por efectos fijos de prueba (para separar los efectos fijos, los de la prueba, de los efectos aleatorios, los del estudiante). Con estos modelos se estima la probabilidad de que una respuesta dada sea correcta en función del número de la prueba (un término lineal del 1 al 5), del género del estudiante (masculino o femenino), del nivel de certeza de la respuesta (alto, medio o bajo) y del total de preguntas de la prueba (ver tabla 2). El género del estudiante también fue usado como término de interacción tanto del número de la prueba como del nivel de certeza de la respuesta.

\section{Resultados}

Este curso comenzó con 43 estudiantes inscritos. En función de ello, en la tabla 2 se puede observar la evolución del número de los que tomó cada prueba, incluyendo el género de los mismos y agregando, como covariable, el número de preguntas que tuvo cada prueba. Además, en la misma tabla se presenta un resumen estadístico descriptivo, tanto de frecuencia absoluta como relativa, de la combinación certeza- 
Tabla 3. Proporción de respuestas correctas

e intervalos de confianza $(a=0,05)$ para el nivel de certeza seleccionado

\begin{tabular}{|lccccccccc|}
\hline & & Total & \multicolumn{3}{c}{ Femenino } & \multicolumn{3}{c|}{ Masculino } \\
& $\begin{array}{c}\text { Lím. } \\
\text { inferior }\end{array}$ & Prop. & $\begin{array}{c}\text { Lím. } \\
\text { superior }\end{array}$ & $\begin{array}{c}\text { Lím. } \\
\text { inferior }\end{array}$ & Prop. & $\begin{array}{c}\text { Lím. } \\
\text { inferior }\end{array}$ & $\begin{array}{c}\text { Lím. } \\
\text { inferior }\end{array}$ & Prop. & $\begin{array}{c}\text { Lím. } \\
\text { inferior }\end{array}$ \\
Alta & 84,7 & 86,4 & 87,9 & 84,9 & 87,0 & 88,9 & 82,5 & 85,4 & 87,9 \\
Media & 57,4 & 60,4 & 63,4 & 57,2 & 60,9 & 64,4 & 55,2 & 60,4 & 65,4 \\
Baja & 41,8 & 45,5 & 49,3 & 40,6 & 45,3 & 50,3 & 40,4 & 46,3 & 52,4 \\
\hline
\end{tabular}

Fuente: Elaboración propia.

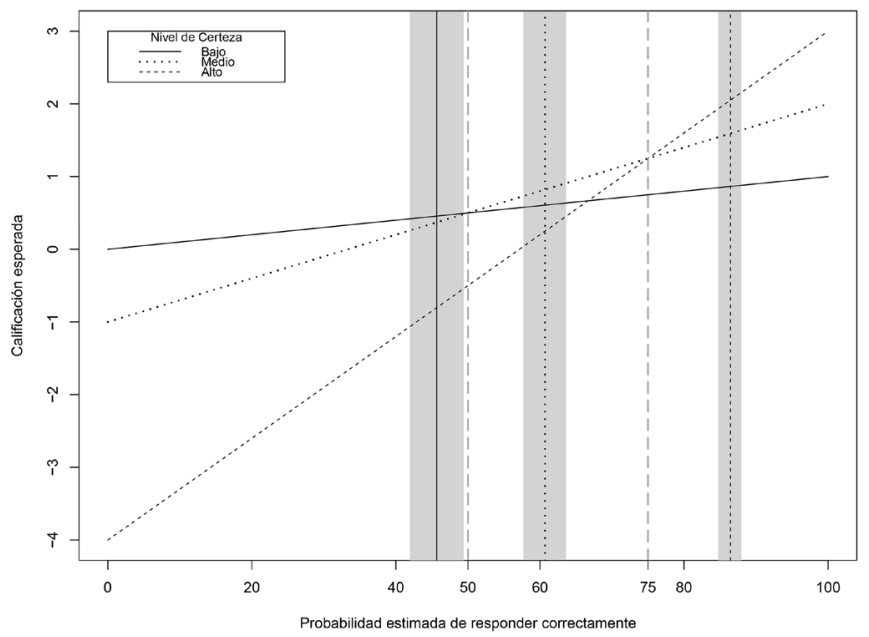

Figura 1. Puntuación esperada de acuerdo con la probabilidad estimada de responder correctamente una pregunta. Las líneas diagonales continua, punteada y discontinua representan la puntuación esperada para un nivel de certeza dado en función de la probabilidad estimada de responder correctamente a una pregunta. Las líneas verticales continua, punteada y discontinua representan la proporción de respuestas correctas para un nivel de certeza dado. Las regiones grises representan sus intervalos de confianza $(a=0,05)$. Las líneas discontinuas grises verticales representan los puntos de corte teóricos de acuerdo con la tabla 1.

resultado en el conjunto de preguntas de cada prueba.

En la tabla 3 se presentan las proporciones de respuestas correctas y los intervalos de confianza $(\alpha=0,05)$ para cada uno de los niveles de certeza establecidos. Los resultados obtenidos son representados gráficamente en la figura 1. De acuerdo con lo descrito por Gardner-Medwin (2006), y ya expuesto en el marco teórico, un estudiante debe ser capaz de identificar adecuadamente la probabilidad de responder correctamente una pregunta, para así poder escoger la certeza que maximice su puntuación esperada. Para el esquema de puntuación presentado en la tabla 1 y figura 1, la primera hipótesis establece que si el estudiante tiene una probabilidad estimada de responder correctamente menor del 50\% (H1: $p<0,5)$, la estrategia óptima de respuesta sería seleccionar un nivel de certeza «bajo», ya que siempre otorga más 
puntos que los demás. Si la probabilidad estimada de responder correctamente es mayor al 50\% (H2a: $p>0,5)$, pero menor al 75\% (H2b: $p<0,75)$, la segunda hipótesis establece que el nivel de certeza que maximiza la calificación esperada del estudiante sería «medio». Finalmente, si la probabilidad estimada de responder correctamente es mayor al 75\% $\left(\mathrm{H}_{3}: p>0,75\right)$, la tercera hipótesis establece que el estudiante debe seleccionar un nivel de certeza «alto».

Estas hipótesis son visualmente comprobadas en la figura 1, ya que tanto la proporción estimada de respuestas correctas para cada nivel de certeza como los límites superior e inferior de los intervalos de confianza calculados se encuentran en las regiones descritas. Además, las tres hipótesis fueron comprobadas estadísticamente al aplicar un test binomial exacto $(\mathrm{H} 1: p<0,5, p$-valor $=0,01043$; H2a: $p>0,5, p$-valor $=$ 1,306e-11; $\mathrm{H} 2 \mathrm{~b}: p<0,75, p$-valor $<2,2 \mathrm{e}-16$; $\mathrm{H}_{3}: p>0,75, p$-valor $\left.<2,2 \mathrm{e}-16\right)$. Aunque no se representan gráficamente, si separamos por géneros los resultados, las hipótesis también fueron comprobadas para el género «femenino» $\left(\mathrm{H}_{1}: p<0,5, p\right.$-valor $=$ o,02875; H2a: $p>0,5, p$-valor $=6,203 \mathrm{e}-9$; H2b: $p<0,75$, $p$-valor $<2,229 \mathrm{e}-16 ; \mathrm{H}_{3}: p$ $>0,75, p$-valor $<2,2 \mathrm{e}-16)$, mientras que casi todas lo fueron para el género «masculino», con excepción de los que han respondido con nivel de certeza «bajo» $\left(\mathrm{H}_{1}: p<\right.$ o,5, $p$-valor $=0,1317$; H2a: $p>0,5, p$-valor $=5,767 \mathrm{e}-5$; H2b: $p<0,75$, $p$-valor $<1,276 \mathrm{e}$ -9; $\mathrm{H}_{3}$ : $p>0,75$, $p$-valor $\left.<6,851 \mathrm{e}-11\right)$. En resumen, los estudiantes fueron capaces de lograr identificar, en promedio, el nivel de certeza adecuado para su probabilidad estimada de responder correctamente una pregunta, excepto para los estudiantes del género «masculino» que han respondido con nivel de certeza «bajo», lo que implica que algunos estudiantes de este grupo fueron más conservadores de lo adecuado al valorar su certeza.

En la tabla 4 se muestran los resultados del modelo logístico de efectos fijos. Se puede apreciar que, al igual que para Gardner-Medwin y Gahan (2006), el género no es una covariable significativa en el modelo, ni aisladamente ni al ser utilizada como término de interacción (se reportan tanto el $p$-valor como los intervalos de confianza teóricos al 95\% de nivel de confianza).

La covariable más importante en este modelo, dado su efecto marginal promedio (Average Marginal Effect), es el haber respondido con certeza alta, ya que incrementa la probabilidad de responder correctamente en un $25 \%$ con respecto a la certeza media, mientras que hacerlo con certeza baja la reduce en un $16 \%$. Se observa, además, un efecto positivo aunque pequeño en el número de prueba, ya que con cada una de ellas que se avanza en el curso se incrementa en un 2,5\% la probabilidad de responder correctamente a una pregunta. No obstante, al acumularlo, se podría decir que un estudiante que toma la quinta prueba tiene cerca de un $10 \%$ más de probabilidad de responder correctamente a una pregunta dada que uno que tomara la primera. Finalmente, cada pregunta que se agregue al cuestionario incrementará en un $0,7 \%$ la probabilidad de responder correctamente. En el caso estudiado, la mayor diferencia 
Tabla 4. Modelo logístico de efectos fijos con términos de interacción.

\begin{tabular}{|c|c|c|c|c|c|}
\hline Efectos Fijos & $\begin{array}{l}\text { Coeficiente } \\
\text { (SE) }\end{array}$ & AME & p-valor & $\begin{array}{l}\text { Int. de Confianza } \\
(a=0,05)\end{array}$ & Sig. \\
\hline Intercepto & $\begin{array}{l}-0,6030 \\
(0.3009)\end{array}$ & & 0,0451 & $(-1,192 ;-0,012)$ & * \\
\hline Prueba & $\begin{array}{l}0,1060 \\
(0,0395)\end{array}$ & 0,0251 & 0,0073 & $(0,029 ; 0,184)$ & $* *$ \\
\hline Masculino & $\begin{array}{l}-0,6381 \\
(0,4958)\end{array}$ & $-0,0090$ & 0,1981 & $(-1,612 ; 0,333)$ & \\
\hline Cert. Baja & $\begin{array}{l}-0,6659 \\
(0,1261)\end{array}$ & $-0,1597$ & $1,28 \mathrm{e}-7$ & $(-0,914 ;-0,419)$ & $* * *$ \\
\hline Cert. Alta & $\begin{array}{l}1,4631 \\
(0,1205)\end{array}$ & 0,2545 & $<2 \mathrm{e}-16$ & $(1,229 ; 1,701)$ & $* * *$ \\
\hline Nro. Preguntas & $\begin{array}{l}0,0343 \\
(0,0133)\end{array}$ & 0,0071 & 0,0101 & $(0,008 ; 0,060)$ & * \\
\hline Prueba: Masculino & $\begin{array}{l}0,0984 \\
(0,0651)\end{array}$ & & 0,1306 & $(-0.029 ; 0,226)$ & \\
\hline Cert. Baja: Masculino & $\begin{array}{l}-0,0205 \\
(0,2109)\end{array}$ & & 0,9225 & $(-0.393 ; 0,433)$ & \\
\hline Cert. Alta: Masculino & $\begin{array}{l}-0.0921 \\
(01994)\end{array}$ & & 0,6442 & $(-0,482 ; 0,300)$ & \\
\hline Nro. Preguntas: Masculino & $\begin{array}{l}0,0153 \\
(0,0220)\end{array}$ & & 0.4858 & $(-0,028 ; 0.058)$ & \\
\hline
\end{tabular}

Nota. Pseudo R-cuadrado $=0,1243443$. AlC $=3675,8$. Códigos de Significancia: $0 * * * 0,001 * * 0,01 * 0,05$ Fuente: Elaboración propia.

entre pruebas es de 10 preguntas (ver tabla 2), por lo que un estudiante tendría un $7 \%$ más de probabilidad de responder correctamente una pregunta dada en la prueba de 25 preguntas que en la de 15 preguntas.

Como contraste a este modelo, en la tabla 5 se pueden apreciar los resultados del modelo logístico mixto con efectos aleatorios, controlando por efectos fijos de prueba. Nuevamente el género no es una variable significativa en el modelo, ni aisladamente ni al ser utilizada como término de interacción (se reportan tanto el $p$-valor como los intervalos de confianza Bootstrap paramétricos con 2000 réplicas al $95 \%$ de nivel de confianza). Los efectos aleatorios, es decir, los asociados a cada estudiante, también fueron significativos en la determinación del intercepto (en este caso se reporta únicamente el intervalo de confianza Bootstrap paramétrico con 2000 réplicas al $95 \%$ de nivel de confianza). Esto implica que hay cambios en los tamaños de los efectos marginales promedios, siendo de los más interesantes el que presenta responder con certeza baja, ya que pasa de un $16 \%$ a un $18 \%$ menos de probabilidad de responder correctamente si selecciona este nivel de certeza, y el asociado al número de preguntas, ya que sube de un $0,7 \%$ a un $0,9 \%$ de acertar una respuesta co- 
Tabla 5. Modelo logístico mixto con efectos aleatorios, controlando por efectos fijos de prueba.

\begin{tabular}{|c|c|c|c|c|c|}
\hline Efectos Aleatorios & Var. & Desv. Est. & & $\begin{array}{l}\text { Int. de Confianza } \\
(a=0,05)\end{array}$ & \\
\hline Sujeto (Intercepto) & 0,1537 & 0,392 & & $(0,237 ; 0,510)$ & \\
\hline Efectos Fijos & $\begin{array}{l}\text { Coef. } \\
\text { (SE) }\end{array}$ & AME & p-Valor & $\begin{array}{l}\text { Int. de Confianza } \\
(a=0,05)\end{array}$ & Sig. \\
\hline Intercepto & $\begin{array}{l}-0,6410 \\
(0.3195)\end{array}$ & & 0,04479 & $(-1,207 ;-0,122)$ & * \\
\hline Prueba & $\begin{array}{l}0,1037 \\
(0,0414)\end{array}$ & 0,0241 & 0,01225 & $(0,028 ; 0,187)$ & * \\
\hline Masculino & $\begin{array}{l}-0,6067 \\
(0,5252)\end{array}$ & 0,0090 & 0,24801 & $(-1,455 ; 0,237)$ & \\
\hline Cert. Baja & $\begin{array}{l}-0,7382 \\
(0,1341)\end{array}$ & $-0,1814$ & $3,72 \mathrm{e}-8$ & $(-1,006 ;-0,471)$ & $* * *$ \\
\hline Cert. Alta & $\begin{array}{l}1,4067 \\
(0,1261)\end{array}$ & 0,2449 & $<2 \mathrm{e}-16$ & $(1,160 ; 1,661)$ & $* * *$ \\
\hline Nro. Preguntas & $\begin{array}{l}0,0394 \\
(0,0138)\end{array}$ & 0,0093 & 0,00421 & $(0,015 ; 0,064)$ & *** \\
\hline Prueba:Masculino & $\begin{array}{l}0,09377 \\
(0,0673)\end{array}$ & & 0,16380 & $(-0.042 ; 0,238)$ & \\
\hline Cert. Baja:Masculino & $\begin{array}{l}-0,0671 \\
(0,2261)\end{array}$ & & 0,76679 & $(-0.500 ; 0,371)$ & \\
\hline Cert. Alta: Masculino & $\begin{array}{l}-0.0502 \\
(0,2093)\end{array}$ & & 0,81033 & $(-0,450 ; 0,377)$ & \\
\hline Nro. Preguntas: Masculino & $\begin{array}{l}0,0135 \\
(0,0226)\end{array}$ & 0,02256 & 0.55017 & $(-0,025 ; 0.051)$ & \\
\hline
\end{tabular}

Nota. R-cuadrado marginal $=0,1489657$. R-cuadrado condicional $=0,1759054 . \mathrm{AIC}=3632,7$. Códigos de Significancia: $0 * * 0,001 * 0,01 * 0,05$. Fuente: Elaboración propia.

rrecta por pregunta adicional. Con este modelo mejoran el R-cuadrado marginal ${ }^{5} \mathrm{y}$ condicional ${ }^{6}$ (Nakagawa y Schielzeth, 2013) en comparación al R-cuadrado ajustado del modelo original, así como el AIC (3632,7 contra 3675,8); una prueba de hipótesis Chi-cuadrado entre los dos modelos muestra diferencias significativas $(p$-valor $=$ $1,88 \mathrm{e}-11)$. Por tanto, se puede apreciar que, usando diferentes medidas de bondad de ajuste, el modelo logístico mixto con efectos aleatorios, controlando por efectos fijos de prueba, es el modelo mejor ajustado a estos datos.

La figura 2 muestra los efectos marginales promedio para el modelo logístico mixto con efectos aleatorios, controlando por efectos fijos de prueba. Se puede observar la probabilidad promedio de responder correctamente a una pregunta dados el género, el nivel de certeza de la respuesta, el número de la prueba y el total de pregun-

5. Varianza explicada por los efectos fijos.

6. Varianza explicada por el modelo logístico mixto. 

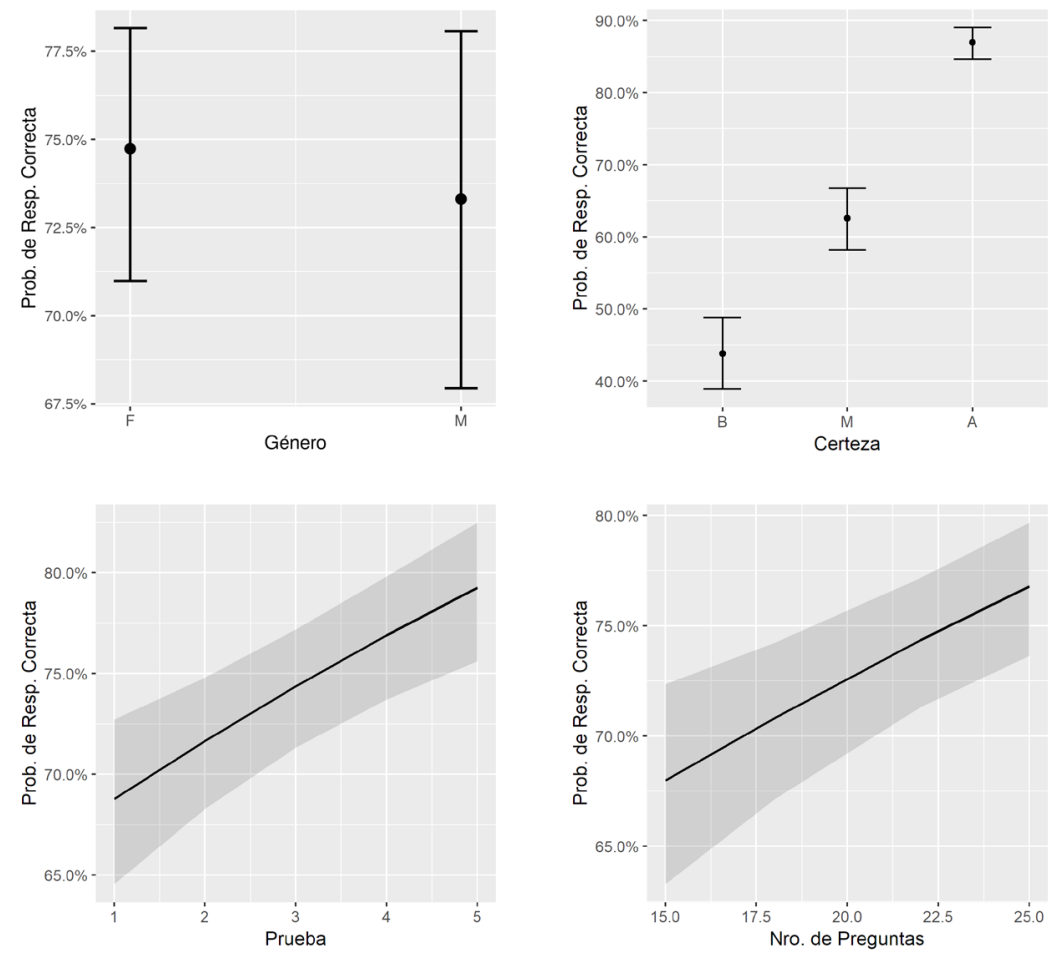

Figura 2. Efectos marginales promedio para el modelo logístico mixto con efectos aleatorios, controlando por efectos fijos de prueba.

tas de la prueba. En cada caso, las variables no mostradas en la gráfica se mantuvieron en el promedio (para las variables continuas) o en la proporción de los casos en los niveles base (para las variables discretas).

En cuanto a la variable de género, aunque en promedio el género femenino tuvo una probabilidad base de responder correctamente a una pregunta dada superior al masculino $(74,7 \%$ contra $73,3 \%)$, las diferencias no son significativas, ya que los intervalos de confianza se solapan. En el caso del nivel de certeza de las respuestas no solo las diferencias son significativas, sino que además la probabilidad crece en torno a $20 \%(43,8 \%$ contra $62,6 \%$ contra $87,0 \%)$ para cada nivel adicional. Nuevamente, queda de manifiesto el proceso de autorregulación de los estudiantes al aplicar el método Certainty-Based Marking.

Con respecto a la prueba, se puede ver que hay una mejoría sustancial desde un $68,8 \%$ de respuestas correctamente acertadas en la primera de ellas a un 79,3\% en la última. Este proceso podría reflejar una especie de «sesgo del sobreviviente», ya que hay estudiantes que abandonan el curso durante las primeras pruebas, algunos de ellos por tener calificaciones bajas (en este caso, 7 estudiantes para un $20 \%$ del curso). No obstante, debido a que entre la cuarta y la quinta prueba hubo un estudiante más 
y aun así mejoraron los resultados, pensamos que al utilizar efectos aleatorios para los estudiantes puede controlarse una parte de este sesgo. Por tanto, se puede pensar que el efecto del proceso de retroalimentación también se evidencia en los resultados.

Finalmente, también queda claro que en una prueba de estilo Certainty-Based Marking debe cuidarse su extensión, ya que el efecto acumulado de ir agregando preguntas es notorio, pasando de un $68 \%$ de respuestas correctamente respondidas para una prueba de 15 preguntas a $76,8 \%$ en una prueba de 25 preguntas. El número ideal deberá ser identificado con sucesivas repeticiones del proceso, variando la cantidad de preguntas y observando sus resultados, buscando que los porcentajes de respuestas correctas no se desvíen significativamente de lo esperado en las hipótesis planteadas en la figura 1.

\section{Discusión y conclusión}

Dados los resultados obtenidos, podemos decir que la aplicación del método Certainty-Based Marking es una alternativa valiosa a la tradicional evaluación oral y escrita usada en los cursos de las escuelas de Derecho. Los fundamentos teóricos que avalan este método de evaluación están sólidamente establecidos y ha sido posible verificarlos empíricamente, como lo hemos hecho en este trabajo.

La autorregulación a través de la autoevaluación, un proceso metacognitivo deseable en todo proceso formativo, es un componente inherente al método CertaintyBased Marking. Nuestros resultados muestran que, a partir de la probabilidad estimada de responder correctamente una pregunta, los estudiantes son capaces de identificar adecuadamente el nivel de certeza que maximiza su calificación esperada. El proceso de justificación de la respuesta que radica en todo sistema de evaluación, aunque no se explicita de manera extensa en el método Certainty-Based Marking, es realizado correctamente por los estudiantes.

De nuestro estudio de regresión logística, podemos destacar que no solo es importante dimensionar generosamente la prueba (en términos de número de preguntas), sino también establecer un proceso de retroalimentación y de evaluación acumulativa, ya que sus efectos son positivos. A cada prueba, tanto el porcentaje de respuestas correctas con factor de certeza alto con respecto al total de respuestas para todos los niveles, como con respecto a las respondidas con certeza alta, fue creciendo a lo largo del año académico. La certeza en la respuesta es, sin duda, el factor más importante entre nuestras covariables, ya que responder con certeza alta incrementa dramáticamente la probabilidad esperada de responder correctamente la pregunta (en promedio un $86 \%$ ).

Finalmente, el hecho de que el género del estudiante no sea una variable significativa para determinar la probabilidad de identificar la respuesta correcta de una pregunta dada es un resultado por demás interesante. La literatura es extensa en 
cuanto al tema de las diferencias existentes en la competencia y la aversión al riesgo con respecto al género de los estudiantes. Parece haber componentes sistémicos que hacen que, en entornos competitivos donde se mezclan personas de ambos géneros, los hombres tiendan a desempeñarse mejor que las mujeres. Si este componente sistémico puede ser reducido o eliminado por el método Certainty-Based Marking en entornos educativos, es algo que no podemos afirmar tajantemente, ya que la muestra es pequeña. Pasa lo contrario con respecto al corpus analizado por Gardner-Medwin y Gahan (2003), que contiene millones de datos. Sería necesario un análisis profundo del sistema pedagógico existente en las escuelas de Derecho chilenas para verificar, como hacen Ho y Kelman (2014) con datos de la Escuela de Derecho de la Universidad de Stanford, que existe una brecha de rendimiento entre géneros y que esta puede ser eliminada, aunque sea parcialmente, con la aplicación de instrumentos de evaluación de este tipo.

\section{Referencias}

Amsterdam, Anthony G. y Jerome Bruner (200o). Minding the law. Boston: Harvard University Press.

Anderson, Lorin y David Krathwohl (2001). A taxonomy for learning, teaching and assessing: A revision of Bloom's taxonomy of educational objectives. Nueva York: Longman.

Azmat, Ghazala y Rosa Ferrer (2017). «Gender gaps in performance: Evidence from young lawyers». Journal of Political Economy, 125 (5): 1306-1355. Disponible en bit. ly/3fGoAkM.

BUtLen, Debora y Philip Winne (1995). «Feedback and self-regulated learning: A theoretical synthesis». Review of Educational Research, 65 (3): 245-281. DOI: 10.2307/1170684.

Coloma Correa, Rodrigo (2005). «El ocaso del profesor Binns: Un ensayo acerca de la enseñanza del derecho en Chile». Ius et Praxis, 11 (1): 133-172. DOI: 10.4067/ So718-00122005000100006.

Dietz, Richard y Sebastiano Moruzzi (2009). Cuts and clouds: Vagueness, its nature and its logic. Oxford: Oxford University Press.

Fernández-Costales MuÑIz, Javier Fernández (2014). «Sobre la metodología docente en la enseñanza de derecho del trabajo y de la seguridad social en el marco del espacio europeo de educación superior». International Journal of Developmental and Educational Psychology, 4 (1) :117-28. DOI 10.1706o/ijodaep.2014.n1.v4.585.

Freire, Paulo (2018). Pedagogía del oprimido. Buenos Aires: Siglo XXI.

GARDNER-MEDwin, Anthony (1995). «Confidence assessment in the teaching of basic science». ALT-J, 3 (1): 80-85. DOI: 10.1080/0968776950030113. 
-. (2006). "Confidence-Based Marking: Towards Deeper Learning and Better Exams». En Cordelia Bryan y Karen Clegg (editoras), Innovative Assessment in Higher Education Bryan (pp. 141-149). Londres: Francis and Taylor.

-. (2019). "Certainty-Based Marking: stimulating thinking and improving objective tests». En Cordelia Bryan and Karen Clegg (editoras), Innovative Assessment in Higher Education (pp. 141-150). 2. ${ }^{\mathrm{a}}$ ed. Londres: Francis and Taylor.

Gardner-Medwin, Anthony R., y Michael Gahan (2003). «Formative and summative confidence-based assessment». En Proceedings of the 7 th CAA Conference (pp. 147-155). Loughborough: Loughborough University.

Gneezy, Uri, Muriel Niederle y Aldo Rustichini (2003). «Performance in competitive environments: Gender differences». The Quarterly Journal of Economics, 118 (3): 1049-1074. DOI: 10.1162/00335530360698496.

GuERrERo, Roberto (1997). «La enseñanza del derecho en Chile: Una visión crítica». Revista Chilena de Derecho, 24 (1): 21-26. Disponible en bit.ly/2ClNJCN.

Guinier, Lani, Michelle Fine y Jane Balin (1994). «Becoming gentlemen: Women's experiences at one Ivy League law school». University of Pennsylvania Law Review, 143 (1): 1-110. Disponible en bit.ly/2YjjgT9.

GneEzy, Uri, Muriel Niederle y Aldo Rustichini (2003). «Performance in competitive environments: Gender differences». The Quarterly Journal of Economics, 118 (3): 1049-1074. Disponible en stanford.io/31dwu1d.

Hattie, John y Helen Timperley (2007). «The power of feedback». Review of educational research, 77 (1): 81-112. DOI: 10.3102/003465430298487.

Ho, Daniel E., y Mark G. Kelman (2014). «Does class size affect the gender gap? A natural experiment in law». The Journal of Legal Studies, 43 (2): 291-321. Disponible en bit.ly/37KnlOC.

LevitT, Steven D., John A. List, Susanne Neckermann y Sally Sadoff (2016). «The behavioralist goes to school: Leveraging behavioral economics to improve educational performance». American Economic Journal: Economic Policy, 8 (4): 183-219. DOI: $10.3386 / \mathrm{w} 18165$.

Martinho, Mariana, Patricia Albergaria-Almeida y José Teixeira Dias (2015). «Cooperation and Competitiveness in Higher Education Science: Does Gender Matter?». Procedia-Social and Behavioral Sciences, 191: 554-558. DOI: 10.1016/j. sbspro.2015.04.569.

MoRIN, Louis-Philippe (2015). «Do men and women respond differently to competition? Evidence from a major education reform». Journal of Labor Economics, 33 (2): 443-491. DOI: 10.1086/678519.

NaKagawa, Shinichi y Holger Schielzeth (2013). «A general and simple method for obtaining R2 from generalized linear mixed-effects models». Methods in ecology and evolution, 4 (2): 133-142. DOI: 10.1111/j.2041-210x.2012.00261. 
PARIS, Scott G. y Peter Winograd (1990). «How metacognition can promote academic learning and instruction». Dimensions of thinking and cognitive instruction, 1: 15-51. Disponible en bit.ly/2YMyWZm.

Rodríguez Ferrara, Mauricio (2004). Acerca de la enseñanza del derecho. 2. ${ }^{a}$ ed. Mérida: CODEPRE.

Squella Narducci, Agustín (2000). «Cultura y cultura jurídica (en el contexto de las revistas jurídicas)». Revista Chilena de Derecho, 27 (4): 655-662. Disponible en bit.ly/3dmchIN.

Sмiтh, Nicholas J.J. (2008). Vagueness and degrees of truth. Oxford: Oxford University Press.

Thurstone, Louis Leon (1919). «A scoring method for mental tests». Psychological Bulletin, 16 (7): 235-240. DOI: 10.1037/hoo69898.

Zimmerman, Barry J. (1989). «Models of Self-Regulated Learning and Academic Achievement». En Barry J. Zimmerman y Dale H. Schunk (editores), Self-regulated learning and academic achievement: Theory, research, and practice (pp. 1-26). Nueva York: Springer-Verlag Publishing.

\section{Agradecimientos}

En primer lugar, agradecemos a Michelle Salazar Mackenna por su invaluable colaboración en la transcripción de los datos. En segundo lugar, al doctor Daniel San Martín y al doctor Tony Gardner-Medwin por su asesoramiento metodológico. Finalmente, nos gustaría agradecer a los revisores anónimos por sus aportes para el mejoramiento de nuestro manuscrito.

\section{Sobre los autores}

Sulan Wong es profesora asociada del Departamento de Ciencias Jurídicas de la Universidad de La Frontera de Chile. Obtuvo su título de abogada en la Universidad de Los Andes de Venezuela. Además, es doctora en Libertades Públicas y Derechos Fundamentales por la Universidade Da Coruña de España. Ha realizado estancias de investigación en la Universitat de Barcelona y tanto en la Université d'Avignon como en el Institut National de Recherche en Informatique et en Automatique de Francia. Es miembro del Centro de Investigación sobre Desafíos Internacionales de la Universidad de La Frontera de Chile. Su principal línea de investigación se centra en las interacciones entre la propiedad intelectual, la ciencia y la tecnología. Su correo electrónico es sulan.wong@ufrontera.cl. (D) https://orcid.org/oooo-0003-1684-0285.

Julio Rojas-Mora es profesor asistente del Departamento de Ingeniería Informática de la Universidad Católica de Temuco de Chile. Obtuvo su título de Ingeniero de Sistemas en la Universidad de Los Andes de Venezuela y un doctorado 
del Departamento de Economía y Organización de Empresas de la Universitat de Barcelona de España. Ha realizado estancias de investigación tanto en la Université d'Avignon como en el Institut National de Recherche en Informatique et en Automatique de Francia. Además, hizo un postdoctorado en el Laboratorio ESPACE del Centre National de la Recherche Scientifique del mismo país. Actualmente es profesor de Métodos Cuantitativos para la Investigación Socioeconómica en Educación del doctorado en Educación de la Universidad Católica de Temuco. Su principal línea de investigación se basa en el estudio de métodos para el apoyo de toma de decisiones en entornos inciertos. Su correo electrónico es jrojas@inf.uct.cl. hittps://orcid. org/0000-0002-0268-8748. 
La Revista Pedagogía Universitaria y Didáctica del Derecho (RPUDD) es una publicación científica semestral que contribuye a la reflexión multidisciplinaria sobre pedagogía universitaria y didáctica del derecho, para la formación y consolidación de esta área de investigación; así como a la difusión de prácticas innovadoras en la enseñanza-aprendizaje del derecho considerando el contexto nacional e internacional. Es una publicación electrónica internacional con una codirección entre Brasil y Chile.

\author{
DIRECTORA \\ María Francisca Elgueta Rosas \\ Universidad de Chile \\ DIRECTOR \\ Renato Duro Dias \\ Universidad Federal de Rio Grande, Brasil \\ SITIO WEB \\ pedagogiaderecho.uchile.cl \\ CORREO ELECTRÓNICO \\ rpedagogia@derecho.uchile.cl \\ LICENCIA DE ESTE ARTÍ́CULO
}

Creative Commons Atribución Compartir Igual 4.0 Internacional

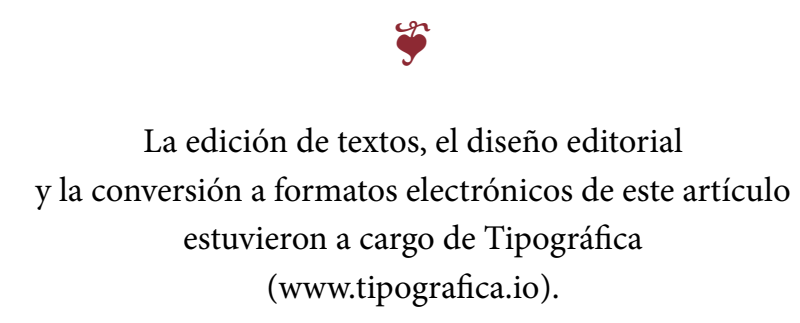

